Acta Crystallographica Section F

Structural Biology

and Crystallization

Communications

ISSN 1744-3091

\section{Jenna $O^{\prime}$ Neill and Anna \\ Roujeinikova*}

Manchester Interdisciplinary Biocentre, Faculty of Life Sciences, University of Manchester, 131 Princess Street, Manchester M1 7DN, England

Correspondence e-mail:

anna.roujeinikova@manchester.ac.uk

Received 5 April 2008

Accepted 27 April 2008

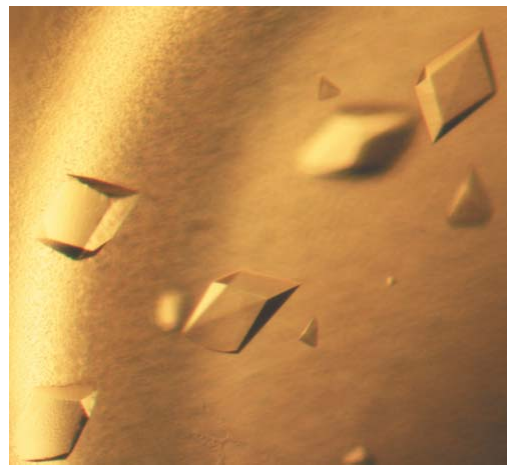

(C) 2008 International Union of Crystallography All rights reserved

\section{Cloning, purification and crystallization of MotB, a stator component of the proton-driven bacterial flagellar motor}

MotB is an essential component of the proton motive force-driven bacterial flagellar motor. It binds to the stress-bearing layer of peptidoglycan in the periplasm, anchoring the MotA/MotB stator unit to the cell wall. Proton flow through the channel formed by the transmembrane helices of MotA and MotB generates the turning force (torque) applied to the rotor. Crystals of recombinant Helicobacter pylori MotB have been obtained by the sitting-drop vapour-diffusion method using ammonium sulfate as a precipitant. These crystals belong to space group $P 4_{1} 2_{1} 2$ or its enantiomorph $P 4_{3} 2{ }_{1} 2$, with unit-cell parameters $a=75.2, b=75.2, c=124.7 \AA$. The asymmetric unit appears to contain one subunit, corresponding to a packing density of $3.4 \AA^{3} \mathrm{Da}^{-1}$. The crystals diffract $\mathrm{X}$-rays to at least $1.8 \AA$ resolution on a synchrotron-radiation source.

\section{Introduction}

Many bacteria swim through fluids by rotating their helical flagella using membrane-embedded molecular rotary motors (DeRosier, 1998; Blair, 2003). Rotation of the bacterial flagellar motor is powered by the proton motive force (pmf) or, in the case of alkalophiles and marine Vibrio species, sodium motive force (Manson et al., 1977; Hirota \& Imae, 1983). In pmf-driven motors, the proton flow through the stator ring formed by the proteins MotA and MotB generates the turning force (torque) applied to the rotor via an as yet unknown molecular mechanism. The proton channel is formed by the transmembrane (TM) helices of MotA and MotB (Zhou et al., 1998; Blair, 2003). MotB has a short N-terminal cytoplasmic segment, a single TM $\alpha$-helix and a large periplasmic domain which shares homology with outer membrane protein A-like domains and is therefore believed to bind to the highly cross-linked stress-bearing layer of peptidoglycan of the cell wall (Chun \& Parkinson, 1988; DeMot \& Vanderleydon, 1994).

Motility by flagellar motor is essential for the virulence of many pathogenic bacteria, including Helicobacter pylori, a Gram-negative bacterium that colonizes the stomachs of roughly $50 \%$ of the world's population (Covacci et al., 1999). H. pylori has a tuft of 4-6 flagella at one end of the cell body that help it drill into the mucus layer of the stomach (Yoshiyama \& Nakazawa, 2000). Motility is required during initial colonization (Eaton et al., 1992) and to attain full infection levels (Ottemann \& Lowenthal, 2002). H. pylori infection has been linked to gastric inflammation and peptic ulcers and is a risk factor for gastric cancer (Uemura et al., 2001). H. pylori possesses a single motB gene (Tomb et al., 1997), in-frame deletion of which creates flagellated nonmotile $H$. pylori mutants that show a reduced ability to infect mice (Ottemann \& Lowenthal, 2002).

In order to elucidate the molecular mechanism by which MotB molecules associate within the stator unit and attach themselves to peptidoglycan, crystallographic studies have been initiated on this protein. The crystallization of a 132-residue C-terminal fragment of H. pylori MotB has recently been reported (Roujeinikova, 2008). In this paper, the cloning, overexpression, purification, crystallization and preliminary X-ray diffraction analysis of the full-length protein are described. 


\section{crystallization communications}

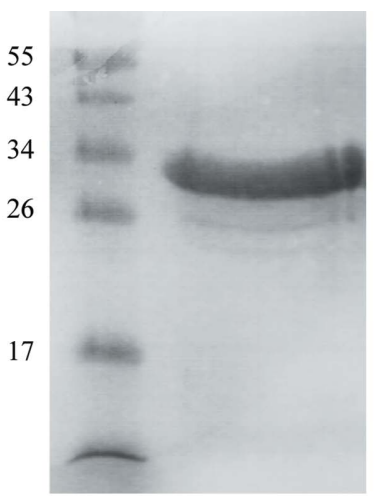

(a)

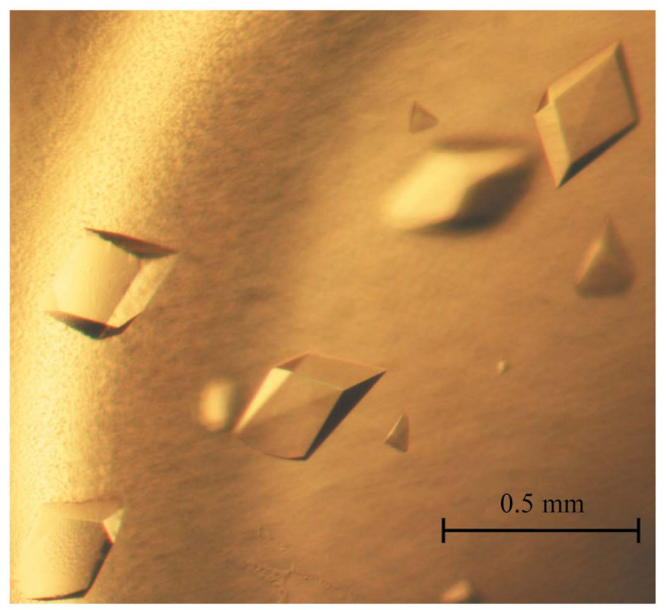

(b)

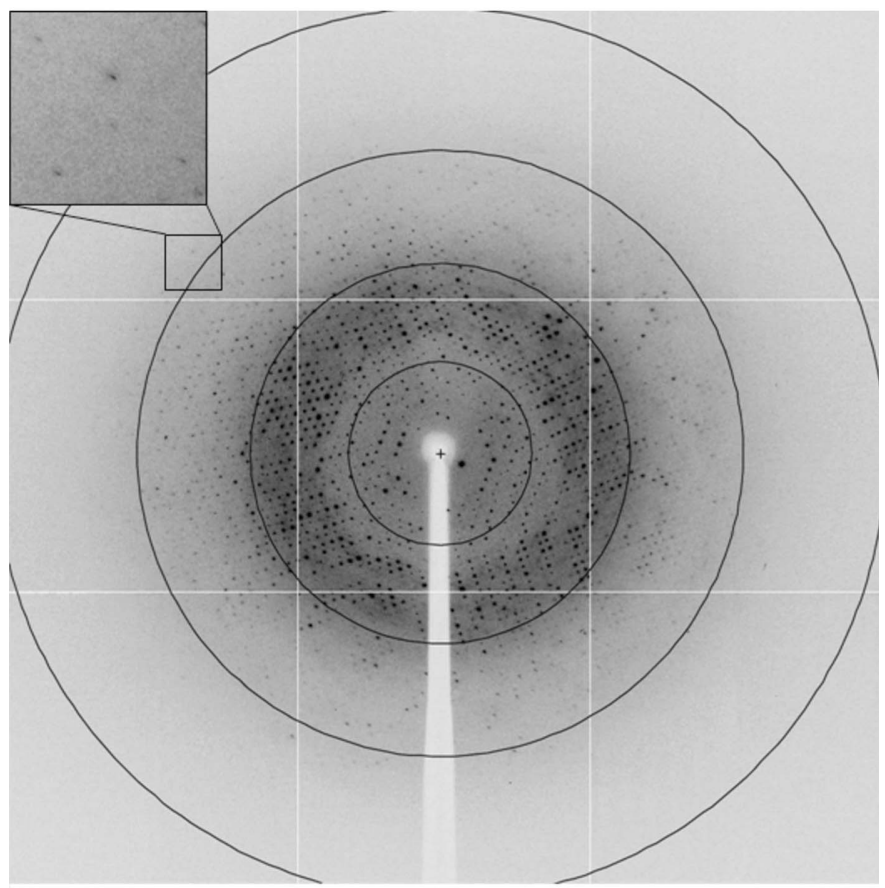

(c)

Figure 1

(a) SDS-PAGE (12\%) analysis of purified recombinant H. pylori MotB (lane 2). Lane 1, PageRuler protein ladder (Fermentas; labelled in kDa). (b) Crystals of H. pylori MotB. (c) A representative $0.5^{\circ}$ oscillation image of data collected from a MotB crystal using an ADSC Quantum 315r CCD detector on station ID14-4, ESRF, France. The magnified rectangle shows diffraction spots at a resolution of $2.0 \AA$ A. The resolution rings lie at $6.2,3.1,2.1$ and $1.6 \AA$.

\section{Materials and methods}

\subsection{Cloning and overexpression}

The gene encoding $H$. pylori MotB (256 residues) was PCRamplified from genomic DNA of $H$. pylori strain 26695 using the HotStar HiFidelity Polymerase kit (Qiagen) and primers CACCGCTAAGAAAAACAAACCCACCGAATG (forward) and TCATTCTTGCTGTTTGTGGGGATTG (reverse). The amplified fragment was cloned into the $\mathrm{pET} 151 / \mathrm{D}-\mathrm{TOPO}$ vector using the TOPO cloning kit (Invitrogen) to produce an expression vector featuring an $\mathrm{N}$-terminal $\mathrm{His}_{6}$ tag followed by the linker GKPIPNPLLGLDSTENLYFQGIDPFT. The expression clone was confirmed by DNA sequencing. The vector was then transformed into Escherichia coli strain BL21 (Novagen). Cells were grown in LB medium containing $100 \mathrm{mg} \mathrm{l}^{-1}$ ampicillin at $310 \mathrm{~K}$ until an $\mathrm{OD}_{600}$ of 0.6 was reached, at which point overexpression of MotB was induced by adding $1 \mathrm{mM}$ IPTG and growth was continued for a further $3 \mathrm{~h}$. The cells were then harvested by centrifugation at $6000 \mathrm{~g}$ for $20 \mathrm{~min}$ at $277 \mathrm{~K}$.

\subsection{Purification}

Cells were lysed by sonication in a buffer containing $50 \mathrm{~m} M$ sodium phosphate $\mathrm{pH} 7.4,100 \mathrm{~m} M \mathrm{NaCl}$ and $1 \mathrm{~m} M$ PMSF. Cell debris was removed by centrifugation at $10000 \mathrm{~g}$ for $20 \mathrm{~min}$. The membrane fraction was isolated by centrifugation at $100000 \mathrm{~g}$ for $2 \mathrm{~h}$ at $277 \mathrm{~K}$. Western blot analysis was carried out on the membrane and soluble fractions using a WesternBreeze Chemiluminescent Detection kit (Invitrogen) with an anti-His-tag antibody (Sigma). The protein was mainly found in the soluble fraction. $\mathrm{NaCl}$ and imidazole were added to the soluble fraction to final concentrations of 500 and $10 \mathrm{mM}$, respectively, after which the supernatant was loaded onto a $5 \mathrm{ml} \mathrm{Hi}$ Trap Chelating HP column (GE Healthcare) pre-washed with buffer $A$ (20 $\mathrm{m} M$ sodium phosphate $\mathrm{pH} 7.4,500 \mathrm{~m} M \mathrm{NaCl}, 10 \mathrm{~m} M$ imidazole, $1 \mathrm{~m} M$ PMSF). The column was washed with 20 column volumes of buffer $B$ (20 m $M$ sodium phosphate pH 7.4, $500 \mathrm{~m} M \mathrm{NaCl}, 80 \mathrm{~m} M$ imidazole) and the protein was eluted with buffer $B$ containing $500 \mathrm{~m} M$ imidazole. The peak fractions were pooled and concentrated to $0.5 \mathrm{ml}$ in a Vivaspin $10000 \mathrm{Da}$ molecular-weight cutoff concentrator and loaded onto a Superdex 75 HiLoad 26/60 gel-filtration column (GE Healthcare) equilibrated with $50 \mathrm{~m} M$ Tris- $\mathrm{HCl} \mathrm{pH}$ 8.0, $200 \mathrm{~m} M \mathrm{NaCl}$. Analysis of the gel-filtration trace (not shown) suggested that MotB is mostly dimeric, with a tendency to form higher oligomers. The fractions containing MotB were identified using SDS-PAGE, pooled and dialysed overnight against $50 \mathrm{mM}$ Tris- $\mathrm{HCl} \mathrm{pH} \mathrm{8.0.} \mathrm{The} \mathrm{purity} \mathrm{of} \mathrm{MotB} \mathrm{was} \mathrm{estimated} \mathrm{to} \mathrm{be} \mathrm{greater}$ than $95 \%$ (Fig. 1a). The protein migrates on SDS-PAGE with an apparent molecular weight of $32 \mathrm{kDa}$, which is close to the value calculated from the amino-acid sequence $(28.8 \mathrm{kDa}$ native protein plus $2.7 \mathrm{kDa} \mathrm{N}$-terminal tag peptide).

\subsection{Crystallization and preliminary $X$-ray analysis}

Prior to crystallization, the protein solution in $50 \mathrm{~m} M$ Tris- $\mathrm{HCl} \mathrm{pH}$ 8.0 was concentrated to $16 \mathrm{mg} \mathrm{ml}^{-1}$ (based on the Bradford assay; Bradford, 1976) and centrifuged for $20 \mathrm{~min}$ at $13000 \mathrm{~g}$ to clarify the solution. Initial screening of crystallization conditions was carried out by the sitting-drop vapour-diffusion method using an automated Phoenix crystallization robot (Art Robbins Instruments) and Crystal Screen, Crystal Screen 2 and PEG/Ion Screen (Hampton Research). The initial crystallization droplets contained $100 \mathrm{nl}$ protein solution mixed with $100 \mathrm{nl}$ reservoir solution and were equilibrated against $100 \mu \mathrm{l}$ reservoir solution in a 96-well Intelliplate (Art Robbins Instruments). Crystals appeared after three weeks from condition No. 
Table 1

Data-collection statistics.

Values in parentheses are for the highest resolution shell.

\begin{tabular}{ll}
\hline Wavelength $(\AA)$ & 1.0 \\
Resolution range $(\AA)$ & $20-1.8(1.9-1.8)$ \\
Completeness $(\%)$ & $91(91)$ \\
Observed reflections & 162389 \\
Unique reflections & 30716 \\
Mean $I / \sigma(I)$ & $16.8(3.5)$ \\
$R_{\text {merge }} \dagger$ & $0.062(0.331)$ \\
\hline
\end{tabular}

$\dagger R_{\text {merge }}=\sum_{h k l} \sum_{i}\left|I_{i}(h k l)-\langle I(h k l)\rangle\right| / \sum_{h k l} \sum_{i} I_{i}(h k l)$, where $I_{i}(h k l)$ is the intensity of the $i$ th observation of reflection $h k l$.

23 of Crystal Screen 2, which contained 1.6 $M$ ammonium sulfate, $100 \mathrm{~m} M$ MES monohydrate $\mathrm{pH} 6.5$ and $10 \%(v / v)$ 1,4-dioxane. Refinement of this condition yielded the optimized composition of the reservoir solution, consisting of $2.1 \mathrm{M}$ ammonium sulfate, $100 \mathrm{~m} M$ MES monohydrate $\mathrm{pH} 6.5$ and $2.5 \%(v / v)$ 1,4-dioxane. Crystals (Fig. $1 b$ ) of maximum dimensions $0.3 \times 0.3 \times 0.4 \mathrm{~mm}$ were obtained using drops containing $3 \mu \mathrm{l}$ protein solution mixed with $3 \mu \mathrm{l}$ reservoir solution and equilibrated against $500 \mu \mathrm{l}$ reservoir solution at $293 \mathrm{~K}$. For data collection, crystals were flash-cooled to $100 \mathrm{~K}$ after soaking in a cryoprotectant solution consisting of the reservoir solution supplemented with $25 \%(v / v)$ glycerol. An X-ray data set was collected from the cryocooled native crystal to $1.8 \AA$ resolution (Fig. 1c) on ESRF beamline ID14-4 (Grenoble, France). A total of 360 images were collected using a $0.5^{\circ}$ oscillation width. All data were processed and scaled using the programs MOSFLM (Leslie, 1992) and SCALA (Collaborative Computational Project, Number 4, 1994). The statistics of data collection are summarized in Table 1.

\section{Results and discussion}

Autoindexing of the diffraction data using MOSFLM is consistent with a primitive tetragonal crystal system, class 422 , with unit-cell parameters $a=75.2, b=75.2, c=124.7 \AA$. The diffraction patterns were examined using the program HKLVIEW (Collaborative Computational Project, Number 4, 1994). Systematic absences were observed along the $a^{*}$ and $c^{*}$ axes, with only reflections with $h=2 n$ and $l=4 n$, respectively, appearing to be present, which suggested that the crystals belong to one of the enantiomorphic space groups $P 4_{1} 22$ or $P 4_{3} 2_{1} 2$. Calculations of the Matthews coefficient (Matthews, 1977) suggested that the asymmetric unit is very likely to contain one molecule, with a $V_{\mathrm{M}}$ value of $3.4 \AA^{3} \mathrm{Da}^{-1}$.

In a search for a suitable model for the molecular-replacement approach, a $B L A S T$ sequence-similarity search against the structures deposited in the Protein Data Bank was carried out. One molecule with a moderate degree of homology to the C-terminal part of MotB has been identified. The 109-residue periplasmic domain of peptidoglycan-associated lipoprotein (PAL) from E. coli (PDB code 2aiz; Parsons et al., 2006) and the putative peptidoglycan-binding domain of MotB (residues 125-256) share 26\% sequence identity in a 102-residue overlap. However, the search model based on the PAL fragment would only constitute $43 \%$ of the full-length MotB structure. Given the limited level of sequence identity, the molecularreplacement approach with this model was not used. Our efforts are currently being directed towards identifying heavy-atom derivatives and the solution of the structure using the multiwavelength anomalous dispersion or multiple isomorphous replacement methods.

The genomic DNA of $H$. pylori strain 26695 was a gift from Dr Nicola High (University of Manchester). We thank Hassan Belrhali at the European Synchrotron Radiation Facility (Grenoble, France) and Patrick Bryant at the X-ray Crystallography Facility (University of Manchester) for assistance with data collection. This work was supported by a Wellcome Trust Research Career Development Fellowship to AR.

\section{References}

Blair, D. F. (2003). FEBS Lett. 545, 86-95.

Bradford, M. M. (1976). Anal. Biochem. 72, 248-254.

Chun, S. Y. \& Parkinson, J. S. (1988). Science, 239, 276-278.

Collaborative Computational Project, Number 4 (1994). Acta Cryst. D50, 760-763.

Covacci, A., Telford, J. L., Del Giudice, G., Parsonnet, J. \& Rappuoli, R. (1999). Science, 284, 1328-1333.

DeMot, R. \& Vanderleydon, J. (1994). Mol. Microbiol. 12, 333-334.

DeRosier, D. J. (1998). Cell, 93, 17-20.

Eaton, K. A., Morgan, D. R. \& Krakowska, S. (1992). J. Med. Microbiol. 37, $123-127$.

Hirota, N. \& Imae, Y. (1983). J. Biol. Chem. 258, 10577-10581.

Leslie, A. G. W. (1992). Jnt CCP4/ESF-EACBM Newsl. Protein Crystallogr. 26.

Manson, M. D., Tedesco, P., Berg, H. C., Harold, F. M. \& Van der Drift, C. (1977). Proc. Natl Acad. Sci. USA, 74, 3060-3064.

Matthews, B. W. (1977). The Proteins, edited by H. Neurath \& R. L. Hill, Vol. 3, pp. 468-477. New York: Academic Press.

Ottemann, K. M. \& Lowenthal, A. C. (2002). Infect. Immun. 70, 1984-1990.

Parsons, L. M., Lin, F. \& Orban, J. (2006). Biochemistry, 45, 2122-2128.

Roujeinikova, A. (2008). Acta Cryst. F64, 277-280.

Tomb, J. F. et al. (1997). Nature (London), 338, 539-547.

Uemura, N., Okamoto, S., Yamamoto, S., Matsumura, N., Yamaguchi, S., Yamakido, M., Taniyama, K., Sasaki, N. \& Schlemper, R. J. (2001). N. Engl. J. Med. 345, 784-789.

Yoshiyama, H. \& Nakazawa, T. (2000). Microbes Infect. 2, 55-60.

Zhou, J., Sharp, L. L., Tang, H. L., Lloyd, S. A., Billings, S., Braun, T. F. \& Blair, D. F. (1998). J. Bacteriol. 180, 2729-2735. 